\title{
Integrated Pest Management with a Mixed Birth Rate for Prey Species
}

\author{
Olcay Akman ${ }^{1}$, Dana Cairns ${ }^{2}$, Timothy D. Comar ${ }^{2,}$, Daniel Hrozencik ${ }^{3}$
}

\begin{abstract}
X. Song and Z. Xiang [7] develop an impulsive differential equations model for a two-prey, one-predator model with stage structure for the predator. They demonstrate the conditions on the impulsive period for which a globally asymptotically stable pest-eradication periodic solution exists, as well as conditions on the impulsive period for which the prey species is permanently maintained under an economically acceptable threshold. We extend their model by including stage structure for both predator and prey and also by adding stochastic elements in the birth rate of the prey. As in [7], we find the conditions under which a globally asymptotically stable pest-eradication periodic solution exists.
\end{abstract}

Keywords: integrated pest management, mixed model, impulsive differential equations

\section{Introduction}

It is well known that a variety of pest species pose a serious health risk to humans and pets, as well as causing great damage to property and crops. For virtually all pest species, biological eradication is biologically impossible or economically infeasible [10]. However, it has been shown that with an integrated pest management (IPM) approach, utilizing combinations of pesticides, predator species, and prey disease, prey species can be controlled at economically and environmentally feasible levels. The IPM approach has been proven superior to either purely biological control or chemical control [7].

A number of recent articles have mathematically modeled a variety of IPM approaches using impulsive differential equations, taking into account, for example, stage structure in the predator species and periodically varying environmental conditions [7]. In the current literature, similar models also have been considered $[9,10]$. These deterministic models assume fixed birth rates for the prey species. As is more realistic in most ecosystems, we consider a random birth rate following a prior distribution with a mean that replaces the fixed birth rate of the previous models considered in $[7,9,10,11,12]$. This approach generalizes the model to accommodate random fluctuations, not just periodic fluctuations, in the birth rate due to environmental and climatic factors. In ecosystems, it is common for the reproductive behavior and fecundity of insect species to be altered by varying environmental and climatic factors such as temperature, light levels, and day length [6]. The stochastic birth rate component in the proposed model accommodates factors such as shortened day length and lower temperatures, which may induce varying levels of egg production [6]. Also, it recognizes that a fixed birth rate really represents an "average" birth rate, which may

\footnotetext{
${ }^{1}$ Department of Mathematics, Illinois State University, Normal, IL

${ }^{2}$ Department of Mathematics, Benedictine University, Lisle, IL

${ }^{3}$ Chicago State University, Chicago, IL

${ }^{*}$ Correspondence: tcomar@ben.edu
} 
produce misleading results as to the resources necessary to ensure a high probability of pest eradication. This is most important in cases in which the population of the prey species is especially sensitive to changes in the potency of the pesticide. This semi-deterministic method is a novel approach. It can be applied to cases in which a priori information is available for birth rate distributions. For instance in cases where small birth pulses are highly probable, a right-skewed probability distribution maybe employed. In fact, even if nothing is known about the prior pulse rates, that is, if any pulse is as likely, then one can use what is called the non-informative prior distribution by assigning equal probabilities under the uniform distribution. The results we obtain will provide information on the necessary values of other parameters that will ensure a high probability of eradication of the prey species under varying birth rates of the prey species.

The present paper is organized as follows: in Section 2, we discuss our impulsive differential equations model, introducing the essential variables and parameters. In Section 3, we use Floquet Theory and results from [7] to establish conditions on the impulsive period for which our pest eradication solution is (i) locally asymptotically stable and (ii) globally asymptotically stable. In Section 4, we introduce a right-skewed distribution for the birth rate parameter $\mathrm{b}$ for the prey species. We present numerical results showing the relationship between the birth rate parameter $\mathrm{b}$ and value of $\mathrm{E}$, the pesticide potency or application effectiveness.

\section{The Mixed Model}

Our deterministic model consists of a prey species with a juvenile class $x_{1}$ and a mature class $x_{2}$, and a predator species with a juvenile class $y_{1}$ and a mature class $y_{2}$. The prey species is born periodically at time intervals of length $T$ via a birth pulse of the form $\frac{\pi b_{1} x_{2}(t)}{q_{1}+x_{1}(t)+x_{2}(t)}+\frac{(1-\pi) b_{2} x_{2}(t)}{q_{2}+x_{1}(t)+x_{2}(t)}$, where $b_{1}, b_{2}, q_{1}$, and $q_{2}$ are positive parameters affecting the birth rate, as considered in the model in [8] and $0 \leq \pi \leq 1$. Our model generalizes the model considered in [1] by considering this mixed birth pulse expression. At the instant of each pulse, the two predator classes $y_{1}$ and $y_{2}$ are augmented by $p_{1}$ and $p_{2}$ respectively. That is, the two predator classes are increased by $p_{1}$ and $p_{2}$ by introducing more of the predator into the population. at the instant of each pulse. Immediately after the births, pesticide is sprayed, which kills a fraction $E$ of both the juvenile and mature prey classes. The prey population is also decreased due to predation by the mature predators only, with parameter $r>0$. The handling time of both $x_{1}$ and $x_{2}$ by the predator is $h$, and the conversion rate of killed prey in excess of what is needed for maintenance into births of new predators is $\lambda$. For instance, if $\lambda=0$, then there is no effect of kills on predator births. Similarly, for very small positive values of $\lambda$, the efficiency of conversion is minimal. This conversion rate expression was also used in the models in [7, 9]. The maturity rates for the prey and predator species are $m_{x}$ and $m_{y}$, respectively. That is, $1 / m_{i}$ is the mean length of the juvenile period. The death rate of the predator is $\mu$. The model equations are given by

$$
\left.\begin{array}{rl}
x_{1}^{\prime}(t) & =-m_{x} x_{1}(t)-r x_{1} y_{2}(t) \\
x_{2}^{\prime}(t) & =m_{x} x_{1}(t)-r x_{2} y_{2}(t) \\
y_{1}^{\prime}(t) & =\frac{\lambda r\left(x_{1}(t)+x_{2}(t)\right) y_{2}(t)}{1+r h\left(x_{1}(t)+x_{2}(t)\right)}-\left(m_{y}+\mu\right) y_{1}(t) \\
y_{2}^{\prime}(t) & =m_{y} y_{1}(t)-\mu y_{2}(t)
\end{array}\right\}, \quad t \neq n T,
$$


This is a system of impulsive differential equations, which we consider only in the biologically meaningful domain $D=\left\{\left(x_{1}, x_{2}, y_{1}, y_{2}\right) \mid x_{1} \geq 0, x_{2} \geq 0, y_{1} \geq 0, y_{2} \geq 0\right\}$. For details on the theory of impulsive differential equations, we refer to the reader to the monograph [4]. For periodic solutions of such impulsive differential equations, see [2]. Furthermore, Lemmas 3.1 and 3.2 provide simple examples of such periodic solutions.

\section{Stability}

We will need the following lemmas for the arguments in this section.

Lemma 3.1 (Song and Xiang [7]). The system

$$
\begin{array}{ll}
u^{\prime}(t)=a-b u(t), & t \neq n T, \\
u_{1}\left(t^{+}\right)=u_{1}(t)+p, & t=n T, \\
u_{1}\left(0^{+}\right)=u_{0} \geq 0 &
\end{array}
$$

has a unique positive, periodic, globally asymptotic solution $\tilde{u}$ with period $T$, given by

$$
\tilde{u}=\frac{a}{b}+\frac{p \exp (-b(t-n T))}{1-\exp (-b T)}, \quad n T<t \leq(n+1) T, n \in \mathbf{N},
$$

and

$$
\tilde{u}\left(0^{+}\right)=\frac{a}{b}+\frac{p}{1-\exp (-b T)} .
$$

For any other solution $u(t)$ of the system, we have $|u(t)-\tilde{u}(t)| \rightarrow 0$ as $t \rightarrow \infty$.

Lemma 3.2 (Song and Xiang [7]). Consider the subsystem

$$
\begin{aligned}
& \left.\begin{array}{l}
y_{1}^{\prime}(t)=-\left(m_{y}+\mu\right) y_{1}(t) \\
y_{2}^{\prime}(t)=m_{y} y_{1}(t)-\mu y_{2}(t)
\end{array}\right\}, \quad t \neq n T, \\
& \left.\begin{array}{l}
y_{1}\left(t^{+}\right)=y_{1}(t)+p_{1} \\
y_{2}\left(t^{+}\right)=y_{2}(t)+p_{2}
\end{array}\right\}, \quad t=n T .
\end{aligned}
$$

The subsystem (3) has the positive, periodic, globally asymptotic solution

$$
\left.\begin{array}{l}
\tilde{y}_{1}(t)=\frac{p_{1} \exp \left(-\left(m_{y}+\mu\right)(t-n T)\right)}{1-\exp \left(-\left(m_{y}+\mu\right) T\right)} \\
\tilde{y}_{2}(t)=\frac{\left(p_{1}+p_{2}\right) \exp (-\mu(t-n T))}{1-\exp (-\mu T)}-\frac{p_{1} \exp \left(-\left(m_{y}+\mu\right)(t-n T)\right)}{1-\exp \left(-\left(m_{y}+\mu\right) T\right)}
\end{array}\right\}, n T<t \leq(n+1) T,
$$

with initial values

$$
\left.\begin{array}{l}
\tilde{y}_{1}\left(0^{+}\right)=\frac{p_{1}}{1-\exp \left(-\left(m_{y}+\mu\right) T\right)} \\
\tilde{y}_{2}\left(0^{+}\right)=\frac{p_{1}+p_{2}}{1-\exp (-\mu T)}-\frac{p_{1}}{1-\exp \left(-\left(m_{y}+\mu\right) T\right)}
\end{array}\right\} .
$$

The following lemma will be needed for the proof of global stability. Its proof is straightforward and similar to the proof of Theorem 5.1 in [11].

Lemma 3.3. There exists a constant $D>0$ such that $x_{1}(t) \leq D, x_{2}(t) \leq D, y_{1}(t) \leq D$, and $y_{2}(t) \leq D$ for each positive solution $\left(x_{1}(t), x_{2}(t), y_{1}(t), y_{2}(t)\right)$ of system (1) and sufficiently large $t$. 
Theorem 3.1. The pest eradication periodic solution $\left(0,0, \tilde{y}_{1}(t), \tilde{y}_{2}(t)\right)$ of system (1) is locally asymptotically stable if

$$
T<\frac{1}{m_{x}} \ln \left(\frac{\left(\left(\frac{\pi b_{1}}{q_{1}}+\frac{(1-\pi) b_{2}}{q_{2}}\right)-1\right)(1-E)+\exp (-r N)(1-E)^{2}}{(1-E)\left(1+\left(\frac{\pi b_{1}}{q_{1}}+\frac{(1-\pi) b_{2}}{q_{2}}\right)\right)-\exp (r N)}\right),
$$

or equivalently, if

$$
\left(\frac{\pi b_{1}}{q_{1}}+\frac{(1-\pi) b_{2}}{q_{2}}\right)<\frac{(1-\exp (-r N)(1-E))\left(1-\exp \left(-m_{x} T-r N\right)(1-E)\right)}{\exp (-r N)\left(1-\exp \left(-m_{x} T\right)\right)(1-E)},
$$

and globally asymptotically stable if

$$
\left(\pi b_{1}+(1-\pi) b_{2}\right)<\frac{1-\exp (-r N)(1-E)}{\exp (-r N)(1-E)},
$$

where

$$
N=\frac{\mu p_{2}+m_{y}\left(p_{1}+p_{2}\right)}{\mu\left(m_{y}+\mu\right)} .
$$

Proof. We first prove that the solution is locally asymptotically stable using Floquet Theory for impulsive differential equations (see [2]). We begin by taking a small amplitude perturbation $\left(u_{1}(t), u_{2}(t), \tilde{y}_{1}(t)+v_{1}(t), \tilde{y}_{2}(t)+v_{2}(t)\right)$ of the pest eradication solution $\left(0,0, \tilde{y}_{1}(t), \tilde{y}_{2}(t)\right)$. Linearizing, we obtain the system

$$
\frac{d \Phi(t)}{d t}=\left[\begin{array}{cccc}
-m_{x}-r \tilde{y}_{2}(t) & 0 & 0 & 0 \\
m_{x} & -r \tilde{y}_{2}(t) & 0 & 0 \\
\lambda r \tilde{y}_{2}(t) & \lambda r \tilde{y}_{2}(t) & -\left(m_{y}+\mu\right) & 0 \\
0 & 0 & m_{y} & -\mu
\end{array}\right] \Phi(t)
$$

where $\Phi(t)$ is the fundamental solution matrix of the system with $\Phi(0)=I$, the identity matrix. The linearization of the pulse behavior is given by

$$
P=\left[\begin{array}{cccc}
1-E & \left(\frac{\pi b_{1}}{q_{1}}+\frac{(1-\pi) b_{2}}{q_{2}}\right)(1-E) & 0 & 0 \\
0 & 1-E & 0 & 0 \\
0 & 0 & 1 & 0 \\
0 & 0 & 0 & 1
\end{array}\right]
$$

Hence, the monodromy matrix of the system is $M=P \Phi(T)$ or

$$
\left[\begin{array}{cccc}
\left(\phi_{1}+b\left(\phi_{2}-\phi_{1}\right)\right)(1-E) & b \phi_{2}(1-E) & 0 & 0 \\
\left(\phi_{2}-\phi_{1}\right)(1-E) & \phi_{2}(1-E) & 0 & 0 \\
* * & * & \exp \left(-\left(m_{y}+\mu\right) T\right) & 0 \\
* * & * & * & \exp (-\mu T)
\end{array}\right],
$$

where

$$
\begin{gathered}
b=\frac{\pi b_{1}}{q_{1}}+\frac{(1-\pi) b_{2}}{q_{2}} \\
\phi_{1}=\exp \left(-\int_{0}^{T}\left(m_{x}+r \tilde{y}_{2}(t)\right) d t\right),
\end{gathered}
$$

and

$$
\phi_{2}=\exp \left(-\int_{0}^{T} r \tilde{y}_{2}(t) d t\right) .
$$

The entries of $M$ marked with "*" are irrelevant in our analysis. By Floquet Theory, the solution is locally asymptotically stable if the absolute values of the eigenvalues of $M$ are less 
than one. This is always the case for the two eigenvalues $\exp \left(-\left(m_{y}+\mu\right) T\right)$ and $\exp (-\mu T)$. The other two eigenvalues are the eigenvalues of the submatrix

$$
\bar{M}=\left[\begin{array}{cc}
\left(\phi_{1}+\left(\frac{\pi b_{1}}{q_{1}}+\frac{(1-\pi) b_{2}}{q_{2}}\right)\left(\phi_{2}-\phi_{1}\right)\right)(1-E) & \left(\frac{\pi b_{1}}{q_{1}}+\frac{(1-\pi) b_{2}}{q_{2}}\right) \phi_{2}(1-E) \\
\left(\phi_{2}-\phi_{1}\right)(1-E) & \phi_{2}(1-E)
\end{array}\right] .
$$

Observe that

$$
\operatorname{det}(\bar{M})=\phi_{1} \phi_{2}(1-E)^{2}>0
$$

and that

$$
\operatorname{trace}(\bar{M})=\left(\phi_{1}+\phi_{2}+\left(\frac{\pi b_{1}}{q_{1}}+\frac{(1-\pi) b_{2}}{q_{2}}\right)\left(\phi_{2}-\phi_{1}\right)\right)(1-E)>0 .
$$

Thus

$$
1+\operatorname{det}(\bar{M})+\operatorname{trace}(\bar{M})>0 .
$$

Therefore the two eigenvalues of the matrix $\bar{M}$ are less than one in absolute value if (see [5] and $[3])$

$$
\operatorname{det}(\bar{M})=\phi_{1} \phi_{2}(1-E)^{2}<1,
$$

and

$$
\begin{aligned}
\operatorname{trace}(\bar{M})-1-\operatorname{det}(\bar{M})= & \left(\phi_{1}+\phi_{2}+\left(\frac{\pi b_{1}}{q_{1}}+\frac{(1-\pi) b_{2}}{q_{2}}\right)\left(\phi_{2}-\phi_{1}\right)\right)(1-E) \\
& -1-\phi_{1} \phi_{2}(1-E)^{2}<0 .
\end{aligned}
$$

Clearly, inequality (10) is always satisfied, and inequality (11) is satisfied by the hypothesis. Hence, the solution is locally asymptotically stable.

We next prove the global attractivity of our solution following the technique in [7]. Choose $\epsilon_{1}>0$ and $\epsilon_{2}>0$ sufficiently small so that

$$
\delta=(1-E)\left(1+\pi b_{1}+(1-\pi) b_{2}\right) \exp \left(r \epsilon_{2} T-r\left(\frac{p_{1}+p_{2}}{\mu}-\frac{p_{1}}{m_{y}+\mu}-\frac{m_{y} \epsilon_{1} T}{\mu}\right)\right)<1 .
$$

We observe that

$$
y_{1}^{\prime}(t) \geq-\left(m_{y}+\mu\right) y_{1}(t)
$$

and consider the following comparison impulsive differential equation:

$$
\begin{array}{ll}
z_{1}^{\prime}(t)=-\left(m_{y}+\mu\right) z_{1}(t), & t \neq n T \\
z_{1}\left(t^{+}\right)=z_{1}(t)+p_{1}, & t=n T \\
z_{1}\left(0^{+}\right)=y_{1}\left(0^{+}\right) \geq 0 . &
\end{array}
$$

By Lemma 3.1, system (12) has a globally asymptotically stable, positive, periodic solution

$$
\tilde{z}_{1}(t)=\frac{p_{1} \exp \left(-\left(m_{y}+\mu\right)(t-n T)\right)}{1-\exp \left(-\left(m_{y}+\mu\right) T\right)}, \quad n T<t \leq(n+1) T .
$$

By the Comparison Lemma for impulsive differential equations (see [4]), we have

$$
y_{1}(t) \geq z_{1}(t)>\tilde{z}_{1}(t)-\epsilon_{1}
$$

From this inequality, we obtain

$$
y_{2}^{\prime}(t) \geq m_{y}\left(\tilde{z}_{1}(t)-\epsilon\right)-\mu y_{2}(t) .
$$

We next consider the comparison system

$$
\begin{array}{ll}
z_{2}^{\prime}(t)=-m_{y}\left(\tilde{z}_{1}(t)-\epsilon\right)-\mu z_{2}(t), \quad t \neq n T, \\
z_{2}\left(t^{+}\right)=z_{2}(t)+p_{2}, & t=n T, \\
z_{2}\left(0^{+}\right)=y_{2}\left(0^{+}\right) \geq 0 . &
\end{array}
$$


By direct calculation, we observe that for $n T<t \leq(n+1) T$,

$$
\tilde{z}_{2}(t)=-\frac{p_{1} \exp \left(-\left(m_{y}+\mu\right)(t-n T)\right)}{1-\exp \left(-\left(m_{y}+\mu\right) T\right)}+\frac{\left(p_{1}+p_{2}\right) \exp (-(\mu(t-n T))}{1-\exp (-\mu T)}-\frac{m_{y} \epsilon_{1}}{\mu}
$$

is a positive, periodic, globally asymptotically stable solution of system (14). Again by the Comparison Lemma, we have

$$
y_{2}(t) \geq z_{2}(t)>\tilde{z}_{2}(t)-\epsilon_{2}
$$

for sufficiently large $t$.

Now let $w(t)=x_{1}(t)+x_{2}(t)$. From the first two equations of system (1), we obtain

$$
w^{\prime}(t) \leq-r w(t)\left(\tilde{z}_{2}(t)-\epsilon_{2}\right)
$$

for $n T<t \leq(n+1) T$, and

$$
w\left(t^{+}\right) \leq w(t)\left(1+\pi b_{1}+(1-\pi) b_{2}\right)(1-E)
$$

for $t=n T$. For $w(t)$, we consider the comparison system

$$
\begin{array}{ll}
z_{3}^{\prime}(t)=-r z_{3}(t)\left(\tilde{z}_{2}(t)-\epsilon_{2}\right), & t \neq n T \\
z_{3}\left(t^{+}\right)=z_{3}(t)\left(1+\pi b_{1}+(1-\pi) b_{2}\right)(1-E), & t=n T \\
z_{3}\left(0^{+}\right)=w\left(0^{+}\right)=x_{1}\left(0^{+}\right)+x_{2}\left(0^{+}\right) \geq 0 . &
\end{array}
$$

By integrating from $t=n T^{+}$to $t=(n+1) T$, we obtain

$$
z_{3}((n+1) T)=z_{3}\left(n T^{+}\right) \exp \left(r \epsilon_{2} T-r \int_{n T}^{(n+1) T} \tilde{z}_{2}(t) d t\right),
$$

where

$$
\int_{n T}^{(n+1) T} \tilde{z}_{2}(t) d t=\frac{\left(p_{1}+p_{2}\right)}{\mu}-\frac{p_{1}}{m_{y}+\mu}-\frac{m_{y} \epsilon_{1} T}{\mu} .
$$

We now obtain the stroboscopic map

$$
\begin{aligned}
z_{3}\left((n+1) T^{+}\right) & =z_{3}\left(n T^{+}\right)\left(1+\pi b_{1}+(1-\pi) b_{2}\right)(1-E) \exp \left(r \epsilon_{2} T-r \int_{n T}^{(n+1) T} \tilde{z}_{2}(t) d t\right) \\
& =z_{3}\left(n T^{+}\right) \delta .
\end{aligned}
$$

Hence, $z_{3}\left(n T^{+}\right)=\delta^{n} z_{3}\left(0^{+}\right)$, and $z_{3}\left(n T^{+}\right) \rightarrow 0$ as $n \rightarrow \infty$. Equation (20) has the unique equilibrium $z_{3}^{*}=0$, which is globally asymptotically stable. Thus, system (18) has the globally asymptotically stable solution $\tilde{z}_{3}(t)=0$. We can conclude that $\lim _{t \rightarrow \infty} w(t)=0$, and hence $\lim _{t \rightarrow \infty} x_{1}(t)=0$ and $\lim _{t \rightarrow \infty} x_{2}(t)=0$, since $x_{1}(t) \geq 0$ and $x_{2}(t) \geq 0$.

We next show that $\lim _{t \rightarrow \infty} y_{1}(t)=0$ and $\lim _{t \rightarrow \infty} y_{2}(t)=0$. For sufficiently small $\epsilon_{3}>0$, there exists $T_{1}>0$ such that $0<x_{1}(t)<\epsilon_{3}$ and $0<x_{2}(t)<\epsilon_{3}$ for all $t>T_{1}$. The function

$$
\frac{\lambda r w(t)}{1+r h w(t)}
$$

is monotonically increasing for $w(t) \geq 0$. By Lemma $3.3, w(t) \leq 2 D$. Let

$$
K=\frac{2 \lambda r \epsilon_{3} D}{1+r h \epsilon_{3}} .
$$

We now have

$$
y_{1}^{\prime}(t) \leq K-\left(m_{y}+\mu\right) y_{1}(t)
$$


Consider the comparison system

$$
\begin{array}{ll}
z_{4}^{\prime}(t)=K-\left(m_{y}+\mu\right) z_{4}(t), & t \neq n T, \\
z_{4}\left(t^{+}\right)=z_{4}(t)+p_{1}, & t=n T, \\
z_{4}\left(0^{+}\right)=y_{1}\left(0^{+}\right) \geq 0 . &
\end{array}
$$

By Lemma 3.1, this comparison system has the positive, periodic, globally asymptotically stable solution

$$
\tilde{z}_{4}(t)=\frac{p_{1} \exp \left(-\left(m_{y}+\mu\right)(t-n T)\right)}{1-\exp \left(-\left(m_{y}+\mu\right) T\right.}+\frac{K}{m_{y}+\mu} .
$$

Hence, for sufficiently small $\epsilon_{4}>0$ and large enough $t$, we have

$$
y_{1}(t) \leq z_{4}(t)<\tilde{z}_{4}(t)+\epsilon_{4} .
$$

From the inequalities (13) and (22), we obtain

$$
\tilde{z}_{1}(t)-\epsilon_{1}<y_{1}(t)<\tilde{z}_{4}(t)+\epsilon_{4}
$$

for sufficiently large $t$. Letting $\epsilon_{1} \rightarrow 0, \epsilon_{3} \rightarrow 0$, and $\epsilon_{4} \rightarrow 0$, we obtain $\tilde{z}_{1}(t) \rightarrow \tilde{y}_{1}(t)$ and $\tilde{z}_{4}(t) \rightarrow \tilde{y}_{1}(t)$ as $t \rightarrow \infty$. Hence, $\lim _{t \rightarrow \infty} y_{1}(t)=\tilde{y}_{1}(t)$.

Using the fourth equation from system (1) and the inequality (22), we obtain the inequality

$$
y_{2}^{\prime}(t) \leq m_{y}\left(\tilde{z}_{4}(t)+\epsilon_{4}\right)-\mu y_{2}(t) .
$$

For this inequality, we consider the comparison system

$$
\begin{array}{ll}
z_{5}^{\prime}(t)=m_{y}\left(\tilde{z}_{4}(t)+\epsilon_{4}\right)-\mu z_{5}(t), & t \neq n T \\
z_{5}\left(t^{+}\right)=z_{5}(t)+p_{2}, & t=n T \\
z_{5}\left(0^{+}\right)=y_{2}\left(0^{+}\right) \geq 0 . &
\end{array}
$$

This system has a periodic, globally asymptotically stable solution

$$
\tilde{z}_{5}(t)=-\frac{p_{1} \exp \left(-\left(m_{y}+\mu\right)(t-n T)\right.}{1-\exp \left(-\left(-m_{y}+\mu\right) T\right)}+\frac{\left(p_{1}+p_{2}\right) \exp (-\mu(t-n T))}{1-\exp (-\mu T)}+\frac{m_{y}}{\mu}\left(\frac{K}{m_{y}+\mu}+\epsilon_{4}\right)
$$

for $n T<t \leq(n+1) T$. By the Comparison Lemma, we have

$$
y_{2}(t) \leq z_{5}(t)<\tilde{z}_{5}(t)+\epsilon_{5}
$$

for sufficiently large $t$. The inequalities (15) and (24) imply that

$$
\tilde{z}_{2}(t)-\epsilon_{2}<y_{2}(t)<\tilde{z}_{5}(t)+\epsilon_{5}
$$

for sufficiently large $t$. Letting $\epsilon_{2} \rightarrow 0, \epsilon_{3} \rightarrow 0$, and $\epsilon_{5} \rightarrow 0$, we obtain $\tilde{z}_{2}(t) \rightarrow \tilde{y}_{2}(t)$ and $\tilde{z}_{5}(t) \rightarrow \tilde{y}_{2}(t)$ as $t \rightarrow \infty$. Hence, $\lim _{t \rightarrow \infty} y_{2}(t)=\tilde{y}_{2}(t)$.

\section{Discussion}

In this paper, we consider an integrated pest management model with two stages for both predator and prey, where prey births occur according to a birth pulse which is a mix of several Beverton-Holt models. We found conditions for global stability of the pest eradication periodic solution. In particular, we express this relationship in terms of an upper bound on a linear combination of the parameters in the birth pulse expression.

It has not escaped our attention that this analysis could be repeated with more than two Beverton-Holt birth pulse terms of the form $\frac{b_{i} x_{2}(t)}{q_{i}+x_{1}(t)+x_{2}(t)}$ so that the entire birth pulse becomes

$$
\sum_{i=1}^{n} \frac{\pi_{i} b_{i} x_{2}(t)}{q_{i}+x_{1}(t)+x_{2}(t)}, \quad \text { where } \quad \sum_{i=1}^{n} \pi_{i}=1
$$


The idea here would be to find the values of the $\pi_{i}$ that produce minimal variance in $E$, the efficacy of the pesticide. This will produce the most stable IPM model in the sense that variations in environmental conditions will require minimal variation in the efficacy of the pesticide to control the pest population. Once we have the values of the $\pi_{i}$ that will minimize the variance in $E$, we can then use data and vary the other parameters to get the best-fit model. This approach offers several advantages. First, we can model a potentially greater variety of birth pulse behavior, which is a priori unknown to the modeler. Second, we have a method for selecting the best, in terms of minimal variance in $E$, from among the different models. Third, once the optimal model has been selected, we can use known methods to fit parameters, giving us a more realistic and accurate model for IPM.

Implementing a mixture of birth-pulse functions with weights $\pi_{i}$ is novel since with this approach, we in fact construct a class of models not just a model whose validity depends on particular data. These weights serve two important purposes. First, they explain the percentage contribution of birth-pulse structures in relation to eradication. Second, they help alleviate the restriction imposed by the lack of information on the birth pulse behavior of pests in question. For instance, in our particular case, an equal weighted mixture of the two functions would indicate that under any choice of the birth-pulse function the overall model would be valid for predicting the level of pesticide efficacy $E$ needed for eradication. Similarly if, say $\pi_{1}=0$ and $\pi_{2}=1$, we can conclude that predicted level of $E$ needed for eradication would be achieved under the second birth pulse function for a given set of free parameters.

\section{References}

[1] O. Akman, T. D. Comar, and D. Hrozencik, 2013. Integrated pest management with a stochastic birth rate for prey species. Frontiers in Neuroscience: Systems Biology 7:141. doi: 10.3389/fnins.2013.00141.

[2] D. D. Bainov and P. S. Simenov, 1993. Impulsive Differential Equations: Periodic Solutions and Applications. New York: Wiley.

[3] S. N. Elaydi, 2005. An Introduction to Difference Equations, 2e. New York, Springer.

[4] V. Lakshmikantham, D. D. Bainov, and P. S. Simeonov, 1989. Theory of Impulsive Differential Equations. Singapore: World Scientific.

[5] J. Li and Y. Yang, 2011. SIR-SVS epidemic models with continuous and impulsive vaccination strategies. Journal of Theoretical Biology, 280, 108-116.

[6] M.D. Paulson, A.I. Houston, J.M. McNamara, and R. J.H. Payne, 2009. Seasonal dispersal of pests: one surge or two? Journal of Evolutionary Biology, 22, 1193-1202.

[7] X. Song and Z. Xiang, 2006. The prey-dependent consumption two-prey one-predator models with stage structure for the predator and impulsive effects. Journal of Theoretical Biology, 242, 683-698.

[8] S. Tang and L. Chen, 2002. Density-dependent birth rate, birth pulse and their population dynamic consequences. Journal of Mathematical Biology, 44, 185-199.

[9] S. Tang et al., 2005. Integrated pest management models and their dynamical behavior. Bulletin of Mathematical Biology, 67, 115-135.

[10] H. Zhang, P. Georgescu, and L. Chen, 2007. An impulsive predator-prey model of integrated pest management. CEU, Department of Mathematics.

[11] Y. Zhang, B. Liu, and L. Chen, 2003. Extinction and permanence of a two-prey onepredator system with impulsive effect. Mathematical Medicine and Biology, 20, 309325. 
[12] Y. Zhang, Z. Xui, and L. Chen, 2005. Dynamic complexity of a two-predator one-prey system with impulsive effect. Chaos, Solitons, and Fractals, 26, 131-139. 